\title{
EL TIEMPO OBJETIVO Y EL SISTEMA DE MUNDOS POSIBLES EN EL TEXTO NARRATIVO
}

\author{
ALFONSO MARTIN JIMÉNEZ \\ (Universidad de Valladolid)
}

Desde los estudios de los formalistas rusos sobre el texto narrativo (Todorov, 1970, 1974: 175; García Berrio, 1973; Erlich, 1974) hasta la concepción actual, que considera una división del tiempo en tiempo objetivo y tiempo subjetivo (Ricoeur, 1987a, 1987b, 1978; Bobes, 1985: 150; Pozuelo Yvancos, 1988: 260 y ss.), así como la inclusión del tempo narrativo en el análisis del relato (Ortega, 1974; Baquero Goyanes, 1974: 234), el estudio del tiempo en la narración ha experimentado un considerable desarrollo, merced a las aportaciones de los diversos autores que han tratado este tema en los últimos años (Weinrich, 1968; Pouillon, 1970; Genette, 1972, 1983; Köck, 1973; Ortega, 1974; Baquero Goyanes, 1970, 1974; Todorov, 1974; Bournef y Ouellet, 1975: 147-150; Segre, 1976, 1985; Ramírez Molas, 1978; Prince, 1982: 26-34, 64-75; Bal, 1985; Ricoeur, 1987a, 1987b, 1978; Bobes, 1985: 147-195; Bobes, 1988; Pozuelo Yvancos, 1988: 260 y ss.).

Hoy en día, la coincidencia es general entre los diversos autores al referirse al tiempo objetivo, que sigue un orden lógico-cronológico en la historia y sufre una serie de transformaciones en el orden de los acontecimientos al plegarse la multiplicidad lineal de dicha estructura a las exigencias de sucesividad unilineal del discurso. En palabras de Todorov, "El problema de la representación del tiempo en el relato se plantea a causa de la diferencia entre la temporalidad de la historia y la del discurso. El tiempo del discurso es, en un cierto sentido, un tiempo lineal, en tanto que el tiempo de la historia es pluridimensional. En la historia, varios acontecimientos pueden desarrollarse al mismo tiempo; pero el discurso debe obligatoriamente ponerlos uno tras otro; una figura compleja se ve proyectada sobre una línea recta. De aquí deriva la necesidad de romper la sucesión "natural" de los acontecimientos, incluso si el autor quisiera seguirla con la mayor fidelidad. Pero la mayor parte de las veces, el autor no trata de recuperar esta sucesión "natural" porque utiliza la deformación temporal con ciertos fines estéticos" (Todorov, 1974: 174). Estas observaciones se refieren a la disposición temporal dentro de una única historia. Sin embargo, y como hace notar Todorov, las formas narrativas más complejas contienen varias historias. Estas historias pueden leerse de varias formas, y los estudios referentes al cuento popular (Propp, 1974) nos ofrecieron ya dos: el encadenamiento (yuxtaposición de diferentes historias, viniendo asegurada la unidad por cierta similitud en la construcción de cada historia) y la intercalación (inclusión de una historia dentro de otra) representando 
estos dos tipos de combinación una proyección rigurosa de las dos relaciones sintácticas fundamentales: la coordinación y la subordinación. Todorov advierte la existencia de un tercer tipo de combinación, que llama de alternancia (Todorov, 1974), consistente en contar dos historias simultáneamente, interrumpiendo ya la una ya la otra para retomarla en la siguiente interrupción, siendo característica de los géneros literarios que han perdido toda unión con la literatura oral.

Cesare Segre, por su parte, tras adoptar la tripartición de discurso (el texto narrativo significante), intriga (el contenido del texto en el mismo orden en el que se presenta) y fábula (los elementos esenciales colocados en un orden lógico-cronológico), afirma que "la diferencia principal entre fábula e intriga radica en el hecho de que la primera respeta la cronología (aunque sea fantástica) de los sucesos y la segunda, en cambio, los mantiene en el orden en el que el escritor los ha descrito" (Segre, 1976: 16).

Gérard Genette parte también de la falta de correspondencia entre el tiempo de la historia y el tiempo del discurso para configurar su sistema analítico (Genette, 1972: 77 182), midiendo la relación entre estos dos distintos tiempos desde tres ejes: 1) Eje de las relaciones entre el orden temporal de sucesión de hechos en la historia y el orden que toman en el relato. 2) Eje de las relaciones de duración: ritmo o rapidez de los hechos en la historia frente al ritmo del discurso. 3) Eje de las relaciones de frecuencia: repetición de hechos en la historia y repeticiones en el discurso. En cuanto al primer apartado, hace notar Genette que lo primero que se advierte al analizar el discurso narrativo es que está repleto de anacronías, de discordancias entre el orden de sucesión en la historia y el orden de sucesión en el discurso. Toda narración presenta una anacronía de orden general, ya que la linealidad del lenguaje obliga a una presentación en orden sucesivo de hechos que quizá son simultáneos. Pero a la vez, toda narración ofrece multitud de anacronías particulares o de detalle. Distingue Genette dos grandes tipos de anacronías: la analepsis, o anacronía retrospectiva hacia el pasado, en la que en una narración primera (presente) se inserta una segunda narración que es temporalmente anterior a ese presente, y la prolepsis, " anacronía prospectiva o anticipadora, hacia el futuro. Con respecto al eje de las relaciones de duración, tampoco se produce una coincidencia o isocronía entre la "duración" de la historia y la del discurso, sino que sólo encontraremos anisocronías en el discurso literario, cuyas principales formas se corresponden con las cuatro formas fundamentales de movimiento narrativo: elipsis (fragmentos de historia que se han suprimido) y pausa descriptiva (pormenorización de algún aspecto concreto de la historia, creándose un tiempo del discurso de mayor duración o ritmo más lento que el de la historia), que son los movimientos extremos, y otros dos movimientos intermedios que se corresponden con lo que un gran número de críticos anglosajones (Lubbock, 1957) llaman sumario o resumen (anisocronía originada cuando se condensa el tiempo de la historia) y escena (intento de ofrecer a los ojos del lector el transcurso de la realidad tal como se produjo). El tercero de los ejes se refiere a las relaciones de frecuencia de hechos en la historia y frecuencia de enunciados narrativos de esos hechos, que pueden reducirse a cuatro formas: relato singulativo (se cuenta una vez lo que ha pasado una vez), relato anáforico (se cuenta $n$ veces lo que ha pasado $n$ veces), relato repetitivo (se cuenta $n$ veces lo que ha pasado una vez) y silepsis (se narra una vez lo que ha pasado $n$ veces).

Aunque el sistema analítico de Genette ha sido adoptado por numerosos autores (Bobes Naves, 1988: 7), no ha sido sin embargo inmune a determinadas observaciones objetadas por la crítica posterior. En este sentido, Paul Ricoeur evidencia hasta qué punto el sistema de análisis temporal de Genette es deudor de una concepción estructuralista que reduce el problema de la temporalidad a los límites del relato mismo (Ricoeur, 1987a, 1987b, 1978), ya que establece que el juego de tiempos en el relato se limita a una relación 
entre la enunciación (tiempo de quien cuenta) y el enunciado (tiempo de lo contado), de forma que identifica el relato con el texto narrativo. Incluso mide la categoría de la duración dentro de la dualidad enunciación-enunciado, sometiendo su análisis al pseudotiempo de la lectura. Procediendo así, Genette omite el tiempo vivido, o tiempo exterior a los límites del acto de producción y recepción del relato, con lo que realiza un estudio de las relaciones temporales interior al mismo texto. Ricoeur, partiendo del pensamiento sobre el tiempo de San Agustín y su idea del triple presente (presente de las cosas presentes, presente de las cosas pasadas y presente de las cosas futuras), y realizando una lectura de la noción aristotélica del mythos (fábula), conjuga estas ideas para postular que narración y temporalidad se autorreclaman: "lo que está últimamente en juego, tanto en la identidad estructural de la función narrativa como en la exigencia de verdad de cualquier obra de este género, es el carácter temporal de la experiencia humana. El mundo desplegado por toda obra narrativa es siempre un mundo temporal. $\mathrm{O},[\ldots]$ el tiempo se hace tiempo humano en cuanto se articula de modo narrativo; a su vez la narración es significativa en la medida en que describe los rasgos de la experiencia temporal" (Ricoeur, 1987a:4l). La correlación que existe entre la actividad de contar una historia y el carácter temporal de la existencia humana no es para Ricoeur puramente accidental, sino que se presenta como una necesidad transcultural, que explica en razón de una triple mímesis: "Tomo como hilo conductor de este análisis de la mediación entre tiempo y narración la articulación [...] entre los tres momentos de la mímesis que llamo mímesis I, mímesis II y mímesis III. Doy por sabido que mímesis /I constituye el eje del análisis; por su función de ruptura, abre el mundo de la composición poética e instituye [...] la literaridad de la obra literaria. Pero mi tesis es que el sentido mismo de la operación de configuración constitutiva de la construcción de la trama resulta de su posición intermedia entre las dos operaciones que yo llamo mímesis $l$ y mímesis $I I I$, y que constituyen 'el antes' y 'el después' de mímesis II" (Ricoeur, 1987a: 118). Por lo tanto, la narratología no puede centrarse únicamente en el campo de la mímesis /I (ordenación narrativa de una historia por medio de la cual una serie de acciones prefiguradas se configuran en una nueva dimensión: la narrativa), sino que ésta sólo puede entenderse como la mediación entre la fuente u origen de la figuración (mímesis I) y la desembocadura o refiguración que ejecuta el lector (mímesis III) (Ricoeur, 1987a; Pozuelo Yvancos, 1988: 261). La tesis de Ricoeur, que abarca el campo filosófico, el lingüístico y el retórico-crítico, plantea que en nuestra experiencia temporal "Seguimos, pues, el paso de un tiempo prefigurado a otro refigurado por la mediación de uno configurado" (Ricoeur, 1987a: 119).

María del Carmen Bobes Naves no margina del análisis temporal la referencia al tiempo vivido, distinto de un tiempo sólamente crónico y que alcanza a la subjetividad o experiencia de un personaje (Bobes, 1985: 147-195; Pozuelo Yvancos, 1988: 265), estableciendo las siguientes posibilidades del uso del tiempo, considerado desde una perspectiva sintáctica, en el discurso novelesco: 1) El tiempo como sucesividad, en progresión que avanza del pasado al futuro, y se manifiesta en fenómenos, naturales o humanos, que siguen una diacronía. 2) El tiempo como orden, que puede ser manipulado en el discurso, pero no en la historia. 3) El tiempo como duración, que se vive en formas diferentes y que es independiente de la medida del tiempo real (Bobes, 1985: 152-153). Como Pozuelo Yvancos hace notar, la principal aportación de Bobes Naves es la incorporación al análisis narrativo de una nueva categoría de la que el sistema genettiano carecía (Pozuelo Yvancos, 1988: 265-266):la del tiempo psicológico o subjetivo, de acuerdo con la idea bergsoniana de "duración", referida al fluir ininterrumpido de los pensamientos de los personajes (Bergson, 1963; Bobes, 1985: 192). 
Cuestión distinta es la del tempo narrativo. Ya Ortega y Gasset, aduciendo que no es el propio argumento de la novela lo que interesa al lector, sino el sentirse impregnado por la presencia de los personajes, había advertido que la novela había de ser un género esencialmente retardatario (Ortega, 1974). Más que la presentación de tramas interesantes, cosa cada vez más difícil de realizar dada la gran cantidad de temas ya tratackos, el novelista moderno ha de idear personajes atractivos, y demorarse en su presentación. Al lector moderno, según Ortega, no le interesa lo que hagan los personajes, sino cómo son los personajes. Esto implica que el autor ha de detenerse en la descripción de la psicología de sus personajes, lo que afecta al tiempo narrativo. Para Ortega, la novela moderna ha de tener, por ello, lo que denomina un tempo lento. Este concepto de Ortega es matizado por Mariano Baquero Goyanes, quien escribe al respecto: "Otra cosa es el tempo novelístico, es decir, el ritmo narrativo, independiente de toda posible angustia temporal. Un mismo asunto puede ser narrado más o menos prolijamente. De la agilidad o lentitud sintáctica -es decir, de la amplitud o brevedad del período-, del manejo del diálogo, de la descripción -según se haga ésta morosamente o con sólo un toque de color-dependerá que el tempo novelístico sea lento o rápido" (Baquero Goyanes, 1974: 234; Paraíso, 1977).

Por lo hasta aquí expuesto, podemos establecer una clasificación de los aspectos concernientes a la temporalidad en el texto narrativo, marcando una diferenciación entre el tiempo y el tempo. El tempo narrativo se refiere al ritmo lento o rápido de la narración. El tiempo puede a su vez subdividirse en tiempo objetivo, o cronológico, como marco en el que se sitúan las acciones externas a los personajes, y tiempo subjetivo, o psicológico, que hace referencia al fluir ininterrumpido de los pensamientos de los personajes y que, al configurarse como duración, es ajeno a los parámetros yue miden el tiempo cronológico.

Centramos a continuación nuestra atención en el estudio del tiempo objetivo, intentando realizar una descripción minuciosa que se traduzca en una mayor precisión explicativa del mismo. Para ello, partimos de la perspectiva que proporciona la Teoría de los Mundos Posibles que, haciéndose eco de las contribuciones filosóficas referidas a los mundos posibles (Prior, 1962; Chismolm, 1967; Hintikka, 1967; Rescher/Parks, 1973; Wittgenstein, 1973; Stalnaker, 1976), e incorporándolas en un modelo teórico-crítico de carácter lingüístico y textual (Albaladejo, 1986a: 50, 1981, 1983, 1984; Chico Rico, 1988; Petöfi, 1979a, 1979b, 1979c, 1979d, 1979e; García Berrio, 1978a, 1978b, 1979a, 1979b, 1980, 1982, 1989), supone un avance con respecto a la narratología de carácter estructural semiológico, estancada en la década de los setenta tras una hipertrofia y crisis de superproducción (García Berrio, 1984: 18; Albaladejo, 1986: 109). Partiendo de la división del texto en los niveles de microestructura, estructura macrosintáctica de transformación, estructura macrosintáctica de base (correspondientes a la división tripartita de Cesare Segre en discurso, intriga y fábula), y enriqueciendo esta clasificación con el apartado subyacente de conjunto referencial (nivel extratextual en el que se enmarcan los elementos referenciales de la realidad que son incorporados al texto tras un proceso de intensionalización) (Albaladejo, 1986a: 65-67; García Berrio/Albaladejo, 1983), la Teoría de los Mundos Posibles (Petöfi, 1979a, 1979b, 1979c; Vaina, 1977; Eco, 1978; Albaladejo, 1986a, 1986b) considera la existencia de un mundo para cada uno de los personajes que forman parte de la trama en el nivel del conjunto referencial y en el resultado de su intensionalización que supone la estructura macrosintáctica de base (Albaladejo, 1986a: 41 42,74 y ss.). Cada mundo de personaje consta a su vez de un submundo articulatorio (o submundo real efectivo) sobre el que se acoplan una serie de submundos no articulatorios (submundos deseado, conocido, fingido, temido...) (Albaladejo, 1986a: 79 y ss.), de manera que el proceso narrativo es consecuencia de la interacción entre unos mundos y otros merced a las relaciones de accesibilidad que entre ellos se producen (Albaladejo, 
1986a: 87, 1986b: 15-16). Cada submundo está constituido por una serie de elementos sucesivos que hacen referencia a las actitudes de experiencia de los personajes, representadas por proposiciones valoradas como verdaderas o como falsas (Albaladejo, 1986a: 78, 174 y ss.). Por consiguiente, el conjunto referencial y la estructura macrosintáctica de base contendrán una serie de líneas paralelas, tantas como mundos de personaje contenga la obra, ordenadas en sucesión temporal lógico-cronológica. Esta pluralidad de líneas temporales debe forzosamente acomodarse a la exigencia de representación unilineal que impone el resultado final del nivel macrosintáctico de transformación.

De esta manera, y tal como expondremos a continuación, la Teoría de los Mundos Posibles, al considerar cada mundo de personaje como una sucesión enumerable de elementos que hacen referencia a las actitudes de experiencia de los personajes, puede proporcionar una explicación minuciosa del tiempo objetivo y sus mecanismos concretos de transformación en el texto narrativo.

Cada uno de los personajes que forman parte del conjunto referencial y de la estructura macrosintáctica de base posee su propia temporalidad, conteniendo estos dos niveles una serie de líneas temporales paralelas en las que se desarrollan los elementos constituyentes de cada mundo de personaje. Aunque la extensión temporal de toda la vida de cada personaje se supone implícita en este nivel, en la realidad de la creación narrativa el autor no suele presentar la totalidad de la vida de los mismos, sino que selecciona los acontecimientos que considera oportuno reflejar, resultando importantes espacios en blanco en la trayectoria vital de cada uno. Por lo tanto, el conjunto referencial y la estructura macrosintáctica de base contienen un conjunto de líneas intermitentes y paralelas en las que se sitúan los elementos dinámicos de la narración (Bobes, 1985: 155), existiendo tantas de estas líneas como personajes formen parte de la obra. Estas líneas temporales son el reflejo de los submundos reales efectivos, o submundos articulatorios, desarrollados en el tiempo objetivo, y sobre ellas se asientan los elementos de los submundos no articulatorios (Albaladejo, 1986a: 80 y ss.), que se producen necesariamente en algún momento del devenir del tiempo objetivo de los personajes. Debido a las relaciones de accesibilidad que tienen lugar entre unos mundos y otros, tales líneas presentan frecuentemente mornentos comunes, resultantes de aquellas situaciones en que dos o mas personajes son protagonistas de un mismo acontecimiento. Estas relaciones de simultaneidad de dos o más personajes se traducen por lo tanto en la existencia de elementos comunes en sus submundos articulatorios.

A este respecto, observamos una clara relación de jerarquización entre los distintos mundos de personaje en función del tiempo objetivo, debido a que el autor suele presentar el tiempo de determinados personajes sólo cuando guarda relación con el tiempo de otro u otros personajes a los que se da más importancia en la narración; es decir, que el tiempo de los personajes secundarios se presenta en relación de simultaneidad con el de otro personaje al que están subordinados temporalmente. Aunque la existencia de toda la vida de los personajes se suponga implícita en este nivel, de hecho sólo son presentados aquellos acontecimientos de su submundo real efectivo que guardan relación de simultaneidad con otros elementos de los submundos reales efectivos de los personajes protagonistas.

Todo texto narrativo presenta una jerarquización de los mundos de personaje en función del tiempo objetivo. Esta jerarquización se produce debido a la necesidad de presentar los tiempos de los personajes secundarios en relación de dependencia con respecto al tiempo de los personajes principales, y posibilita la presentación exclusiva del tiempo de los personajes que el autor quiere resaltar. En definitiva, el autor organiza el 
tiempo de su relato distribuyéndolo en los mundos de los personajes que forman parte de él, expresando de manera explícita una mayor extensión del tiempo de los personajes que le interesa remarcar e incluyendo la presentación del tiempo de los personajes menos importantes cuando éste gira en torno a un momento que guarda relación de simultaneidad con respecto al de los principales. Este entramado jerárquico temporal que posibilita la narración y que se refleja en la existencia de elementos comunes en los submundos reales efectivos de los personajes, tanto en el nivel del conjunto referencial como en el de la estructura macrosintáctica de base, se verá reproducido en la estructura macrosintáctica de transformación y en la microestructura.

La pluralidad de líneas temporales del conjunto referencial y de la estructurá macrosintáctica de base debe, por otra parte, resolverse en la única línea temporal característica del resultado del nivel macrosintáctico de transformación. Para explicar esta confrontación entre las múltiples líneas temporales de la fábula y la única línea de presemáación de los hechos del sujeto, postulamos la existencia de un Doble Mecanismo Transformativo Temporal (DoMeTT), que actúa en dos fases sucesivas. La activación de su primera fase da como resultado una ordenación temporal concreta de cada mundo de personaje que puede no coincidir con la ordenación temporal que dicho mundo presentaba en el nivel de la fábula. En esta primera fase se mantiene por lo tanto la pluralidad de líneas temporales característica del conjunto referencial y de la estructura macrosintáctica de base, si bien cada una de tales líneas puede poseer una ordenación diferente de los hechos. En cada una de estas líneas temporales se insertan los elementos de todos los submundos de personaje, de manera que cada elemento de los submundos no articulatorios (deseado, conocido, creído, fingido...) se inserta en el lugar de la temporalidad que le corresponde del submundo real efectivo (Submundo articulatorio). Por lo tanto, cada mundo completo de personaje adquiere una sucesividad unilineal, estableciéndose tantas de estas líneas como mundos de personaje contenga el texto.

Supongamos que un texto consta de $n$ mundos de personaje, y que cada uno de estos mundos presenta una determinada ordenación de sus submundos en la fábula:

Mundo 1:

a) submundo real efectivo (r.e.): elementos $1,2,3, \ldots$ n.

b) submundo conocido (c.): elementos $1,2, \ldots \mathrm{n}$.

Mundo 2:

a) suhmundo real efectivo (r.e.): elementos $1,2,3, \ldots$ n.

b) submundo conocido (c.): elementos $1,2, \ldots n$.

Miundo $n$ :

a) submundo real efectivo (r.e.): elementos $1,2,3, \ldots$ n.

b) submundo conocido (c.): elementos $1,2, \ldots n$.

Al activarse la primera fase del DoMeTT, se obtiene como resultado una sola línea temporal para cada mundo, con una ordenación que puede no corresponder con la de la fábula, manteniéndose tantas líneas como mundos $n$ haya de personajes. Por ejemplo:

Mundo 1 (M1): elementos 1r.e., 2c., 3r.e., 2r.e., 1c., ...n. 
Mundo 2 (M2): elementos 2r.e., 1c., 1r.e., 2c., 3r.e., ...n.

Mundo $n(\mathrm{M} n)$ : elementos 1r.e., 1c., 2r.e., 2c., 3r.e., ...n.

Una vez obtenidas estas líneas se produce la activación de la segunda fase del DoMeTT. Por medio de ella, se elige el orden de disposición de los elementos de las múltiples líneas en una única línea temporal, pero manteniendo obligatoriamente para cada mundo el orden resultante de la fase anterior. Se realiza en esta segunda fase, pues, una combinación de la presentación de los mundos, pero no del orden de los elementos que forman cada mundo (pues éste ya se estableció en la fase anterior). El resultado de esta segunda fase configura la organización temporal definitiva que se plasmará literariamente en la microestructura. El resultado podría ser, por ejemplo:

Elementos Ir.e.MI, 2r.e.M2, 2c:MI, Ir.e.Mn, 1c. $\mathrm{Mn}$, 3r.e.MI ...n.

O cualquier otra combinación posible que no presente un orden distinto del resultante para cada línea en la fase anterior (si abstraemos en este ejemplo los elementossubrayados-resultantes del Mundo 1 (M1) en la fase anterior, su ordenación sigue siendo la misma: 1r.e., ...2c., ...3r.e., ..., y de igual forma ocurre en cada mundo).

Al activarse la primera fase del DoMeTT puede producirse, además, la elisión de determinados elementos del mundo de cada personaje que aparecían explícitos en la fábula y se suponen implícitos en este nivel. Esta elisión de elementos puede afectar tanto a los constituyentes del submundo real efectivo como a los de los submundos no articulatorios, comenzando a gestarse de esta forma el aligeramiento de elementos de la fábula que posibilita la plasmación efectiva del resultado del sujeto en la microestructura. Puede producirse también en este nivel la repetición de determinados elementos del mundo de cada personaje, si el autor decide reproducir su inclusión por cualquier razón (simple insistencia, intensificación estética, explicación de un elemento insinuado cori anterioridad...). Tal repetición conlleva necesariamente una transformación temporal en el orden de los elementos del mundo del personaje del que se trate. Asímismo, determinadas transformaciones temporales pueden verse asociadas a la cuestión del punto de vista narrativo en esta primera fase del DoMeTT, tanto en lo referente a la focalización como a la voz narradora (Friedmann, 1969; Pouillon, 1970; Todorov, 1971: 99 y ss., 1974: 177 y ss.; Genette, 1972: 20 y ss., 1983: 77-83; Bal, 1977; Pozuelo Yvancos, 1984). Si an mismo elemento del mundo de un personaje es presentado desde el punto de vista cie varios personajes focalizadores, la repetición de este elemento supone necesariamente una transformación temporal en la ordenación de su propio mundo. También pueder producirse transformaciones temporales obligatorias en los casos en los que el narrador forme parte de la fábula -narrador homodiegético, en terminología de Genette- (Genette, 1983: 77-83) y desconozca determinados elementos de su propio submundo real efectivo -caso de la situación narradora "yo-protagonista" de Norman Friedmann- o del mundo del personaje protagonista -situación narradora "yo-testigo"- (Friedmann, 1969), ya que estas informaciones podrán ser presentadas por el narrador omnisciente o por otro personaje narrador en otro momento del discurso, con la consiguiente modificación temporal.

Al activarse la segunda fase del DoMeTT se produce asimismo la elisión de aigunos elementos de los submundos reales efectivos de los personajes subordinados a otros temporalmente. En efecto, si dos o más personajes protagonizan un mismo acto, éste se 
ve reflejado en la fábula en un elemento común a los mundos de todos ellos; sin embargo, en el sujeto, y debido a la activación de la segunda fase del DoMeTT, tal acontecimiento podrá figurar una sola vez, como constituyente del mundo de personaje que ocupe un nivel más alto en el orden jerárquico temporal. Aunque en la fábula existan elementos explícitos en los mundos de todos los personajes referidos al acontecimiento que comparten, resultaría redundante reflejar en el sujeto el mismo hecho repetidamente como perteneciente al mundo de todos los personajes que lo protagonizan. Por ello el autor suele limitarse a exponer el elemento del submundo articulatorio del personaje que ocupa más alto nivel en el escalafón jerárquico temporal, elidiendo los elementos de los submundos articulatorios de los personajes subordinados, que sin embargo se suponen implícitos en el elemento reflejado del personaje principal. Se observa así con absoluta claridad en este nivel la existencia de una jerarquización de los mundos de personaje en función del tiempo objetivo, necesaria para que se realice el aligeramiento de elementos que posibilita una exposición económica y eficaz de los contenidos. Si esta jerarquización se evidencia en esta fase es porque existe previamente en los niveles subyacentes de estructura macrosintáctica de base y conjunto referencial.

La jerarquización temporal hace referencia específica a los submundos reales efectivos de los personajes, y no a sus submundos no articulatorios (deseado, conocido, cré́do...). Esto es debido a que los elementos de los submundos no articulatorios son exclusivos del mundo de cada personaje, y no pueden producirse relaciones de simultaneidad entre los elementos de los submundos no articulatorios de unos personajes y otros. Por lo tanto, todos los elementos de los submundos no articulatorios que permanecían explicitos tras la primera fase del DoMeTT seguirán permaneciendo explícitos tras la activación de sus segunda fase. No ocurre lo mismo con gran parte de los elementos de los submundos reales efectivos de los personajes subordinados a otros temporalmente, que se ven muchas veces elididos en favor de la presentación única del elemento del mundo del personaje del que dependen. Sin embargo, si el autor decidiera presentar varias veces en la microestructura un mismo acontecimiento desde el punto de vista de cada uno de los personajes que lo protagonizan, los elementos referidos a este acontecimiento en los mundos de los diversos personajes partícipes en la acción no serían elididos, apareciendo en el resultado final de este nivel como constituyentes de los mundos de tales personajes. Por otra parte, la elección del punto de vista narrativo (voz y focalización) no condiciona en esta segunda fase del DoMeTT la aparición de nuevas transformaciones temporales en cada mundo, ya que estas se produjeron en la primera fase.

La postulación de la existencia del DoMe'TT obedece a una concepción teórica, ya que en la realidad de la producción literaria el autor no es consciente de su aplicación en dos fases sucesivas, como tampoco lo es el lector en su proceso analítico inverso de interpretación. Su enunciación, sin embargo, permite explicar minuciosamente cómo la pluralidad de líneas temporales de la fábula se resuelve en la única línea temporal resultante del sujeto.

La jerarquización de los mundos de personaje en función del tiempo objetivo se revela como uno de los mecanismos inherentes a todo género narrativo, hasta el punto de que sin su concurso no sería posible construir un texto narrativo eficaz. El aligeramiento progresivo de los elementos del nivel macrosintáctico de base en el nivel macrosintáctico de transformación, posibilitado por la existencia de una jerarquización temporal y llevado a cabo por la activación del doble mecanismo transformativo temporal, permite una economía de la expresión en el nivel de la microestructura que hace factible la narración. Corresponde al lector, siguiendo un proceso analítico inverso al proceso creativo sintético efectuado por el autor, recomponer la totalidad de los elementos subyacentes en el texto 
a partir de la microestructura que se le presenta. Cuanto más complejas sean las transformaciones efectuadas en el nivel del sujeto, mayor será la atención que el lector deberá prestar para obtener la configuración subyacente de la fábula, por lo que la activación del doble mecanismo transformativo temporal presenta magníficas posibilidades al autor de cara a atraer el interés del lector hacia su texto.

\section{Referencias bibliograficas}

Albaladejo Mayordomo, T. (1981), "Aspectos del análisis formal de textos", en Revista Española de lingüística, 11, 1, pp.117-160.

Albaladejo Mayordomo, Tomás (1983), "Componente pragmático, componente de representación y modelo lingüístico-textual", en Lingua e Stile, 18, I, pp.3-46.

Albaladejo Mayordomo, Tomás (1984), "Estructura de sentido, representación textual semántico-intensional y tópico textuatl", en: Anales de la Universidad de Murcia, Letras, XL.III, 1-2, 1984-85, pp. 265-284.

Albaladejo Mayordomo, Tomás (1986a), Teoría de los munlos posibles y macroestructura narrativa, Alicante, Universidad de Alicante.

Albaladejo Mayordomo, Tomás (1986b), "La organización de mundos en el texto narrativo. Análisis de un cuento de 'El Conde Lucanor'", en: Revista de Literatura, XLVIII, 95, pp. 5-18.

Bal, M. (1977), "Narration et focalisation", en: Poétique, 29, pp.107-127.

Bal, M. (1985), Teoría de la narrativa. Una introducción a la narratología (Vers. esp. Madrid, Cátedra).

Baquero Goyanes, J. M. (1970), Estructuras de la novela actual, Barcelona, Planeta.

Baquero Goyanes, J. M. (1974), "Tiempo y 'tempo' en la novela", en: G. Gullón y A. Gullón (eds.), Teoría de la novela, Madrid, Taurus, pp. 231-242.

Bergson, H. (1963), "Ensayo sobre los datos inmediatos", en: Obras escogidas, Madrid, Aguilar.

Bobes Naves, J. (1988), Las novelas "caribes" de Francisco Ayala, Kassel, Universidad de Oviedo-Edition Reichenberger.

Bobes Naves, M.C. (1985), Teoría General de la Novela. Semiología de "La Regenta", Madrid, Gredos.

Bournef, R. Ouellet, R. (1985), La novela (Vers. esp. Barcelona,Ariel).

Chico Rico, F. (1988), Pragmática y construcción literaria. Discurso retórico y disclarso narrativo, Alicante, Universidad de Alicante.

Chismolm, R.M. (1967), "Identity Through Possible Worlds: Some Questions", en: Noûs, 1,1, pp. $1-8$.

Eco, U. (1978), "Possible Worlds and Text Pragmatics: 'Un drame bien parisien'", en: Versus, 19-20, pp. 5-72.

Erlich, V. (1974), El formalismo ruso (Vers. esp. Barcelona, Seix Barral).

Friedmann, N. (1969), "Point of View. The Development of a Critical Concept", en: R. Murray, The Novel. Essays on Criticism, New Jersey.

García Berrio, A. (1973), Significado actual del formalismo ruso, Barcelona, Planeta.

García Berrio, A. (1978a), "Lingüística del texto y texto lírico. La tradición textual como contexto", en: Revista española de Lingüistica, 8, 1, pp. 19-75.

García Berrio, A. (1978b), "Tipología textual de los sonetos clásicos españoles sobre el 'carpe diem'", en: Dispositio, 3, pp. 243-293.

García Berrio, A. (1979a), "Situación de la teoría textual. (La teoría de J. S. Petöfi en el 
marco de la lingüística del texto)", en: J.S. Petöfi/A. García Berrio, Lingüística del texto y crítica literaria, Madrid, Comunicación, pp. 243-264.

García Berrio, A. (1979b), "Lingüística, literaridad/poeticidad. (Gramática, pragmática, texto)", en: 1616. Anuario de la Sociedad Española de Literatura General y Comparada, 2, pp. 125-170.

García Berrio, A. (1980), "Construcción textual en los sonetos de Lope de Vega. Tipología del macrocomponente sintáctico", en: Revista de Filología Española, 60, 1978-1980, pp. 23-157.

García Berrio, A. (1982), "Problemas de la determinación del tópico textual. El soneto en el Siglo de Oro", en: Anales de Literatura Española de la Universidad de Alicante, 1, pp. 135-205.

García Berrio, A. (1984), "Retórica como ciencia de la expresividad. (Presupuestos para una Retórica General)", en: Estudios de Lingüistica de la Universidad de Alicante, 2 , pp. 7-59.

García Berrio, A. (1989), Teoría de la Literatura (La construcción del significado poético), Madrid, Cátedra.

Genette, G. (1972), Figures III, París, Seuil.

Genette, G. (1983), Nouveau discours du récit, París, Seuil.

Hintikka, J. (1967), "Individuals, Possible Worlds and Epistemic Logic", en: Noâs, 1,1, pp. 33-62.

Kück, W. (1973), "Time and Text: Towards an Adequate Heuristics", en: J.S. Petöfi/H. Rieser (eds.), Studies in Text Grammar, Dordrecht, Reidel, pp. 113-204.

Lubbock, P. (1957), The Craft of Fiction, New York, The Viking Press.

Ortega y Gasset, J. (1974), "Ideas sobre la novela", en: G. Gullón/A. Gullón (eds.), Teoría de la Novela, Madrid, Taurus, pp. 29-64.

Paraíso del Leal, I. (1977), Teoría del ritmo de la prosa, Madrid, Cupsa.

Petöfi, J.S. (1979a), "Una teoría formal semiótica como teoría integrada del lenguaje natural", en J.S. Petöfi/A. García Berrio (1979), pp. 127-145.

Petöfi, J. S. (1979b), "Estructura y función del componente gramatical de la teoría de la estructura del texto y de la estructura del mundo", en: J.S. Petöfi/A. García Berrio (1979), pp. 147-189.

Petöfi, J. S. (1979c), "La representación del texto y el léxico como red semántica", en: J.S. Petöfi/A. García Berrio (1979), pp. 215-242.

Petöfi, J. S. (1979d), "Léxico, conocimiento enciclopédico, teoría del texto", en: J.S. Petöfi/A. García Berrio (1979), pp. 191-213.

Petöfi, J. S. (1979e), "La teoría lógico-semántica de las lenguas naturales como teoría textual", en J.S. Petöfi/A. García Berrio (1979), pp. 99-125.

Petöfi, J.S./García Berrio, A. (1979), Lingüística del texto y Crítica literaria, Madrid, Comunicación.

Pouillon, J. (1970), Tiempo y novela (Vers. esp. Buenos Aires, Paidós).

Pozuelo Yvancos, J.M. (1984), "Focalización y estructura textual: la capilla de Brandeso en

la 'Sonata de Otoño", en: Estudios de Lingüistica, 2, pp. 251-271.

Pozuelo Yvancos, J. M. (1988), Teoría del lenguaje literario, Madrid, Cátedra.

Prince, G. (1982), Narratology. The Form and Functioning of Narrative, Berlin, Mouton Publishers.

Prior, A.N. (1962), "Possible Worlds", en: Philosophical Quarterly, 12, 46, pp.36-43.

Propp, V. (1974), Morfología del cuento (Vers. esp. Madrid, Fundamentos).

Ramírez Molas, P. (1978), Tiempo y narración. Enfoques de la temporalidad en Borges, Carpentier, Cortázar y García Márquez,Madrid, Gredos. 
Rescher, N./Parks, Z. (1973), "Possible Individuals, Trans-World Identity and Quantified Modal Logic", en: Noûs, 7, 4, pp. 330-350.

Ricoeur, P. (1978), Temps et récit III, París, Seuil.

Ricoeur, P. (1987a), Tiempo y narración I (Vers. esp. Madrid, Cristiandad).

Ricoeur, P. (1987b), Tiempo y narración II (Vers. esp. Madrid, Cristiandad).

Segre, C. (1976), Las estructuras y el tiempo (Vers. esp. Barcelona, Planeta).

Segre, C. (1985), Principios de análisis del texto literario (Vers. esp. Barcelona, Crítica).

Stalnaker, R.C. (1976), "Possible Worlds", en: Nô̂s, 10, pp. 65-75.

Todorov, T. (1970), Teoria de la literatura de los formalistas rusos (Vers. esp. Buenos Aires, Signos).

Todorov, T. (1971), Literatura y significación (Vers. esp. Barcelona, Planeta).

Todorov, T. (1974), "Las categorías del relato literario", en: VV.AA., Análisis estructural del relato, "Comunicaciones", 8 (Vers. esp. Buenos Aires, Mundo Contemporáneo).

Vaina, L. (1977), "Introduction: les 'mondes possibles' du texte", en: Versus, 17, pp. 5-72.

Weinrich, H. (1968), EStructura y función de los tiempos en el lenguaje (Vers. esp. Madrid, Gredos).

Wittgenstein, L. (1973), Tractatus logico-philosophicus (Vers. esp. Madrid, Alianza). 